\title{
The clinical prognostic value of PD-L1 after concurrent chemoradiotherapy in Chinese nasopharyngeal carcinoma patients
}

\author{
Guoxian Long ${ }^{1 \#}$, Xiaoyu Li $^{1 \#}$, Lin Yang ${ }^{1}$, Jing Zhao ${ }^{1}$, Xiang Lu $^{2}$, Heng Wang ${ }^{2}$, Jia Song ${ }^{2}$, Qi Mei ${ }^{1}$, \\ Guangyuan $\mathrm{Hu}^{1}$
}

${ }^{1}$ Department of Oncology, Tongji Hospital, Tongji Medical College, Huazhong University of Science and Technology, Wuhan, China; ${ }^{2}$ Department of Otolaryngology-Head and Neck Surgery, Tongji Hospital, Tongji Medical College, Huazhong University of Science and Technology, Wuhan, China

Contributions: (I) Conception and design: Q Mei, G Hu, G Long, X Li; (II) Administrative support: Q Mei, L Yang; (III) Provision of study materials or patients: G Long, L Yang, J Zhao; (IV) Collection and assembly of data: X Li, H Wang, J Song; (V) Data analysis and interpretation: G Long, X Li; (VI) Manuscript writing: All authors; (VII) Final approval of manuscript: All authors.

\#These authors contributed equally to this work.

Correspondence to: Qi Mei; Guangyuan Hu. Department of Oncology, Tongji Hospital, Tongji Medical College, Huazhong University of Science and Technology, Wuhan 430030, China. Email: borismq@hotmail.com; h.g.y.121@163.com.

Background: Although immune checkpoint inhibitor (ICI) therapy has revolutionized the treatment of nasopharyngeal carcinoma (NPC), it is still the second- or third-line treatment after the failure of radiotherapy or chemotherapy. In this study, we aimed to investigate the impact of concurrent chemoradiotherapy (CCRT) on programmed death-ligand 1 (PD-L1) protein expression in NPC patients.

Methods: We enrolled 24 NPC patients treated with intensity-modulated radiation therapy (IMRT) combined with cisplatin CCRT. PD-L1 expression was evaluated by immunohistochemistry, and nextgeneration sequencing and annotation were performed to determine the genetic alteration after CCRT.

Results: Our results showed that patients with a high expression of PD-L1 were more inclined to a complete response (CR) to chemoradiotherapy, as opposed to a partial response $(\mathrm{PR})(\mathrm{P}<0.05)$. Moreover, the mean values of the tumor mutation burden (TMB) and the tumor neoantigen burden (TNB) in the PDL1 positive group were significantly lower than that of the PD-L1 negative group in our cohort.

Conclusions: We confirmed that the TMB and TNB may be potential clinical indicators in NPC treatment, and PD-L1 expression may be a clinical biomarker in NPC chemoradiotherapy. Finally, through next-generation sequencing and annotation, we found that the most frequent driver gene mutations in NPC were TET2, TP53, and MAPK.

Keywords: Programmed death-ligand 1 (PD-L1); nasopharyngeal carcinoma (NPC); biomarker; tumor mutation burden (TMB); tumor neoantigen burden (TNB)

Submitted Aug 05, 2021. Accepted for publication Nov 03, 2021.

doi: $10.21037 / \mathrm{atm}-21-5175$

View this article at: https://dx.doi.org/10.21037/atm-21-5175

\section{Introduction}

The prevalence of nasopharyngeal carcinoma (NPC) is rare, and is characterized by distinctive geographic distribution, with a particular prevalence in Southeast Asia and China $(1,2)$. Due to the special anatomical location of the nasopharynx, most NPC patients are classified as advanced NPC at first diagnosis (3). Despite the fact that NPC is particularly sensitive to radiotherapy and chemotherapy, local recurrence and distant metastasis are still the major causes of NPC treatment failure, accounting 
for approximately $5-15 \%$ and $15-30 \%$, respectively (4-6). In 2012, the high tumor metastasis rate of NPC resulted in a global incidence of mortality of approximately 50,000 cases (7).

With the application of intensity-modulated radiation therapy (IMRT), the local control of NPC and the overall survival (OS) of NPC patients have improved markedly. First-line platinum-based chemotherapy is the most typically used therapeutic regimen in the treatment of NPC. IMRT combined with cisplatin concurrent chemoradiotherapy (CCRT) is the recommended standard treatment for patients with locoregionally advanced nasopharyngeal carcinoma (LANPC) by the National Comprehensive Cancer Network Guidelines (NCCN 2017 version) (8). However, even with an optimized treatment, approximately $5-15 \%$ of NPC patients have local recurrence, and approximately $15-30 \%$ of NPC patients experience failure due to distant metastasis (9).

Notably, programmed death-ligand 1 (PD-L1) and programmed death-1 (PD-1) inhibitors exhibit encouraging outcomes in head and neck cancer, hepatocellular carcinoma, non-small-cell lung cancer, and NPC (10-12). Despite the emergence of immune checkpoint inhibitors (ICIs) as a promising therapy for various cancers, most NPC patients do not respond positively to ICI therapy, which may explain why ICI therapy is applied as a secondor third-line treatment after the failure of platinum-based chemotherapy (4).

ICI treatments benefit some patients with metastatic cancers, but predictive biomarkers are urgently needed. PDL1 has been shown to be overexpressed in numerous cancer cell types and is associated with different clinical outcomes, either better or worse, depending on the tumor category (12-15). PD-L1 is one of the immune-checkpoint molecules that regulates type $1 \mathrm{~T}$ helper (Th1) immune responses and mediates cancer immune evasion. The expression of PD$\mathrm{L} 1$ has been reported to be a prognostic predictor and is also a predictive biomarker of the response to PD-1/PD-L1 inhibitors in different cancer, including NPC. Some reports have verified that the upregulation of PD-L1 expression in NPC is associated with a poor OS and may be used as a new prognostic factor for NPC (13). However, there are some controversies about the prognostic value of $\mathrm{PD}$ L1 expression on specific types of cancer, such as breast cancer, non-small cell lung cancer, and NPC $(9,16,17)$. The prognostic value of PD-L1 expression in NPC remains unclear. Cao et al. demonstrated that tumor PDL1 expression is inversely associated with poor outcomes in patients with NPC (18). However, Kawaguchi et al. reported that the patients who carried positive PD-L1 expression tumor cells or immune cells manifested remarkable rates of advantageous progression-free survival and OS (19). Based on the conflicting results, convincing evidence to evaluate the prognostic value of PD-L1 expression in the NPC is needed.

Herein, we aim to evaluate the alteration of PD$\mathrm{L} 1$ protein expression and the correlation between $\mathrm{PD}$ L1 expression to CCRT in 24 matched (pre-and postchemoradiotherapy) Chinese NPC patients. In this study, we evaluate the Landscape of somatic mutations of NPC patients by Next-generation sequencing. We also analyzed the TMB and TNB value difference between PD-L1 positive NPC patients and PD-L1 negative NPC patients. We present the following article in accordance with the REMARK reporting checklist (available at https://dx.doi. org/10.21037/atm-21-5175).

\section{Methods}

\section{Patients}

Tumor samples from 24 NPC patients (none immune checkpoint inhibitor therapy) before and after CCRT were collected in the Tongji Hospital from 2018 to 2019. Informed consent was obtained from all patients, and all patients underwent pathological reconfirmation by a senior pathologist and pre-treatment imaging. Patients with any other severe diseases, such hematological diseases or contagious diseases, were excluded from this study. A serial physical examination, including chest $\mathrm{X}$-ray, bone scan, and abdominal ultrasound, were applied to evaluate NPC metastases. The tumor samples [formalin-fixed and paraffin-embedded (FFPE) tissue specimens] were sent for hematoxylin and eosin (HE) staining, histopathological, and immunohistochemistry examination. All procedures performed in this study involving human participants were under the Declaration of Helsinki (revised in 2013). This study was approved by the Ethics Committee of Tongji Hospital of Tongji Medical College, Huazhong University of Science and Technology, China. All methods were carried out their relevant guidelines and regulations. Written informed consent was obtained from all study participants.

\section{Histological subtype classification}

All HE images were identified by one otolaryngologist and 
two experienced pathologists using the same microscope (IX71, Olympus, Tokyo, Japan), and histological subtypes were divided according to the World Health Organization tumor classification standard [2015].

\section{Immunobistochemistry}

FFPE tissues obtained from surgeries were divided into 3 for subsequent testing. The central position of each FFPE tumor tissue was selected to verify contain tumor cells by H\&E staining. Subsequently, FFPE tissues were continuously sectioned with a thickness of $4 \mu \mathrm{m}$. After deparaffinization, the rabbit monoclonal PD-L1 antibody (E1L3N, 1:800, cell signaling technology, Danvers, MA, USA) was used for immunohistochemical staining. For imagery of the antigen, a peroxidase-labeled secondary antibody (DAKO, 22c3) was used.

\section{PD-L1 scoring}

As there were no established criteria for PD-L1 in NPC, the percentage of positive PD-L1 expression in FFPF tissues (tumor proportion score, TPS) was independently evaluated by three experienced pathologists using the same microscope (IX71, Olympus, Tokyo, Japan), and the average TPSs from each examiner per case was acquired. IHC was utilized for PD-L1 expression was assessed using a scoring system with several cut-offs $(1 \%, 5 \%, 10 \%, 15 \%$, to $100 \%)$ as previously described. Sections with less than $1 \%$ tumor staining were regarded as negative $(20,21)$.

\section{Next-generation sequencing and annotation}

Genomic DNA was exacted from FFPE tissue sections, and then sonicated to $200 \mathrm{bp}$ fragments. The Roche SeqCap EZ Exome V3 (Roche NimbleGen Inc., Basel, Switzerland) and TruePrep DNA Library Prep Kit V2 for Illumina (\#TD501, Vazyme, Nanjing, China) were used to capture target DNA, and Illumina HiSeq machines were used to generate pairedend sequence data. Subsequently, alignment was performed using binary alignment/map (BWA), and PCR duplication was sort and exclude with The Genome Analysis Toolkit (GATK, http://www.broadinstitute.org/gsa/wiki/index.php/ The_Genome_Analysis_Toolkit) (22). Copy number variants (CNVs) from whole-exome sequencing data were detected by CNVKIT (https://github.com/etal/cnvkit) (23). Somatic mutations were converted to MAF format and visualized by $\mathrm{R}$ package maftools (https://bioconductor.org/packages/ release/bioc/vignettes/maftools/inst/doc/maftools.html).

\section{Statistical analysis}

The PD-L1 scores were evaluated as continuous variables, and statistical analyses were performed using SPSS version 22.0 (Statistical Package for the Social Sciences, SPSS Inc., Chicago, IL, USA). Clinicopathologic variables of the PDL1 high group and PD-L1 low group were compared using the chi-squared or Fisher's exact tests for nominal variables. Since our study involved a small sample size, all of the clinical-related statistical analyses were considered to be significant when $\mathrm{P}<0.05$, without a multiple correction test.

\section{Results}

\section{Patient characteristics}

We selected 24 patients, including 18 males and 6 females. The median age of patients was 47 years (range, 19-64 years). Eleven patients were Epstein-Barr virus (EBV)-positive. Among the 24 patients, 1 (4.17\%), 7 (29.17\%), and 16 (66.67\%) patients were clinical stage II, III, and IVA, respectively. IMRT was applied for all patients; 13 patients $(54.17 \%)$ were combined with docetaxel (DOC) + nedaplatin (NDP) + 5-fluorouracil (5FU) concurrent, 2 (8.33\%) were combined with DOC + cisplatin (DDP) + capsaicin (CAP) concurrent, $1(4.17 \%)$ was combined with DOC + NDP + CAP concurrent, 1 (4.17\%) was combined with DOC + DDP + 5FU concurrent, 5 (20.83\%) were combined with DOC + NDP concurrent, and $2(8.33 \%)$ were combined with gemcitabine (GEM) + DDP concurrent. The patients' characteristics are illustrated in Table 1 and Table S1.

\section{Histopathological examination of advanced patients}

Representative pre and post-treatment immunohistochemistry staining of PD-L1 in locoregionally advanced NPC biopsies is presented in Figure 1A-1F. Representative pre and posttreatment $\mathrm{HE}$ staining images of tumor biopsies are depicted in Figure 1G-1L. The central cells of the slice had remarkable heteromorphism, disorganized arrangement, large deep staining of nuclei, and regular nuclear fission in the pretreatment biopsy images (Figure $1 G, 1 I, 1 \mathrm{~K}$ ). Necrosis and increased interstitial cell could be seen in the post-treatment biopsy images (Figure 1H,17,1L), which may be partly due to interstitial inflammatory cell infiltration during CCRT. 
Table 1 Baseline patient characteristics of 24 NPC patients

\begin{tabular}{|c|c|}
\hline Characteristic & N (\%) \\
\hline Median age in years [range] & $47[19-64]$ \\
\hline Male/female & $18(75.0) / 6(25.0)$ \\
\hline \multicolumn{2}{|l|}{ T-classification } \\
\hline $\mathrm{T} 1$ & $1(4.17)$ \\
\hline $\mathrm{T} 2$ & $1(4.17)$ \\
\hline T3 & $10(41.67)$ \\
\hline $\mathrm{T} 4$ & $12(50.0)$ \\
\hline \multicolumn{2}{|l|}{$\mathrm{N}$-classification } \\
\hline NO & $0(0.0)$ \\
\hline N1 & $7(29.17)$ \\
\hline N2 & $11(45.83)$ \\
\hline N3 & $5(20.83)$ \\
\hline N4 & $0(0.0)$ \\
\hline \multicolumn{2}{|l|}{ Clinical stage } \\
\hline I & $0(0.0)$ \\
\hline II & $1(4.17)$ \\
\hline III & $7(29.17)$ \\
\hline IVA & $16(66.67)$ \\
\hline \multicolumn{2}{|l|}{ Chemotherapy regimen } \\
\hline $\mathrm{DOC}+\mathrm{NDP}+5 \mathrm{FU}$ & $13(54.17)$ \\
\hline $\mathrm{DOC}+\mathrm{DDP}+\mathrm{CAP}$ & $2(8.33)$ \\
\hline $\mathrm{DOC}+\mathrm{NDP}+\mathrm{CAP}$ & $1(4.17)$ \\
\hline $\mathrm{DOC}+\mathrm{DDP}+5 \mathrm{FU}$ & $1(4.17)$ \\
\hline $\mathrm{DOC}+\mathrm{NDP}$ & $5(20.83)$ \\
\hline GEM + DDP & $2(8.33)$ \\
\hline \multicolumn{2}{|c|}{ PD-L1 expression level before treatment } \\
\hline Negative (<1\%) & $9(37.50)$ \\
\hline$<1 \%$ and $<5 \%$ & $11(47.83)$ \\
\hline$>5 \%$ & $4(17.39)$ \\
\hline
\end{tabular}

NPC, nasopharyngeal carcinoma; DOC, docetaxel; NDP, nedaplatin; 5FU, 5-fluorouracil; DDP, cisplatin; CAP, capsaicin; GEM, gemcitabine.

\section{Correlation between PD-L1 immunohistochemistry and response to CCRT}

We analyzed the correlation between the pre- and posttreatment PD-L1 expression and the therapeutic response in TPS. Eight patients $(33.33 \%)$ had a partial response (PR) to chemoradiotherapy, 11 patients $(45.83 \%)$ had a complete response (CR) to chemoradiotherapy, and two patients (8.33\%) had stable disease (SD) (Figure 2A). Coincidentally, the correlation between the pre- and post-treatment PD$\mathrm{L} 1$ expression and the therapeutic response in combined positive score (CPS) was the same as in TPS (Figure 2A,2B). The data of two patients $(8.33 \%)$ was not available (NA). Clearly, patients with a high expression of PD-L1 were more inclined to a $\mathrm{CR}$, as opposed to a $\mathrm{PR}(\mathrm{P}<0.05)$. Thus, we can infer that high levels of PD-L1 expression result in a better therapeutic outcome from CCRT.

\section{Expression of PD-L1 before and after CCRT}

Among all patients, nine (37.50\%) had PD-L1 negative expression, nine $(37.50 \%)$ had $<5 \%$ PD-L1 positive expression, and four (17.39) had $>5 \%$ PD-L1 positive expression (Table 1). After CCRT, the PD-L1 expression in TPS was unchanged in 7 patients (29\%), increased in 10 patients $(42 \%)$, and decreased in 7 patients $(29 \%)$ (Figure 2C). Interestingly, the PD-L1 expression in the CPS was unchanged in four patients (16.67\%), increased in eight patients $(33.33 \%)$, and decreased in 12 patients $(50.00 \%)$ (Figure 2D). These results illustrated that NPC patients might have PD-L1 expression alteration after CCRT.

\section{Landscape of somatic mutations in NPC patients}

Whole exome sequencing (WES) was performed on preCCRT paired samples from 18 NPC patients to assess the somatic alternation in advanced NPC samples. The average coverage depth for samples was 196x. There were 8,514 mutation events in 1,778 genes from cancer and adjacent tissues, including the most frequent driver gene mutations in NPC (TET2, TP53, and MAPK). Detailed gene mutation information of the 18 NPC patients are shown in available online: https://cdn.amegroups.cn/static/ public/atm-21-5175-01.xlsx. In our cohort, the mean value of the tumor neoantigen burden (TNB) and tumor mutation burden (TMB, mutations/Mb) in the PD-L1 positive group was significantly lower than the PD-L1 negative group (Figure $3 A, 3 B$ ). The top 10 mutated genes are depicted in Figure $3 C$. Moreover, we identified significantly mutated genes (SMGs) in NPC mutation cohort. The oncoplot of the top 20 (ARID1B, MED12, CEBPA, TEKT4, TET2, etc.) mostly mutated genes showed distribution diversity among each NPC sample (Figure 3D). 

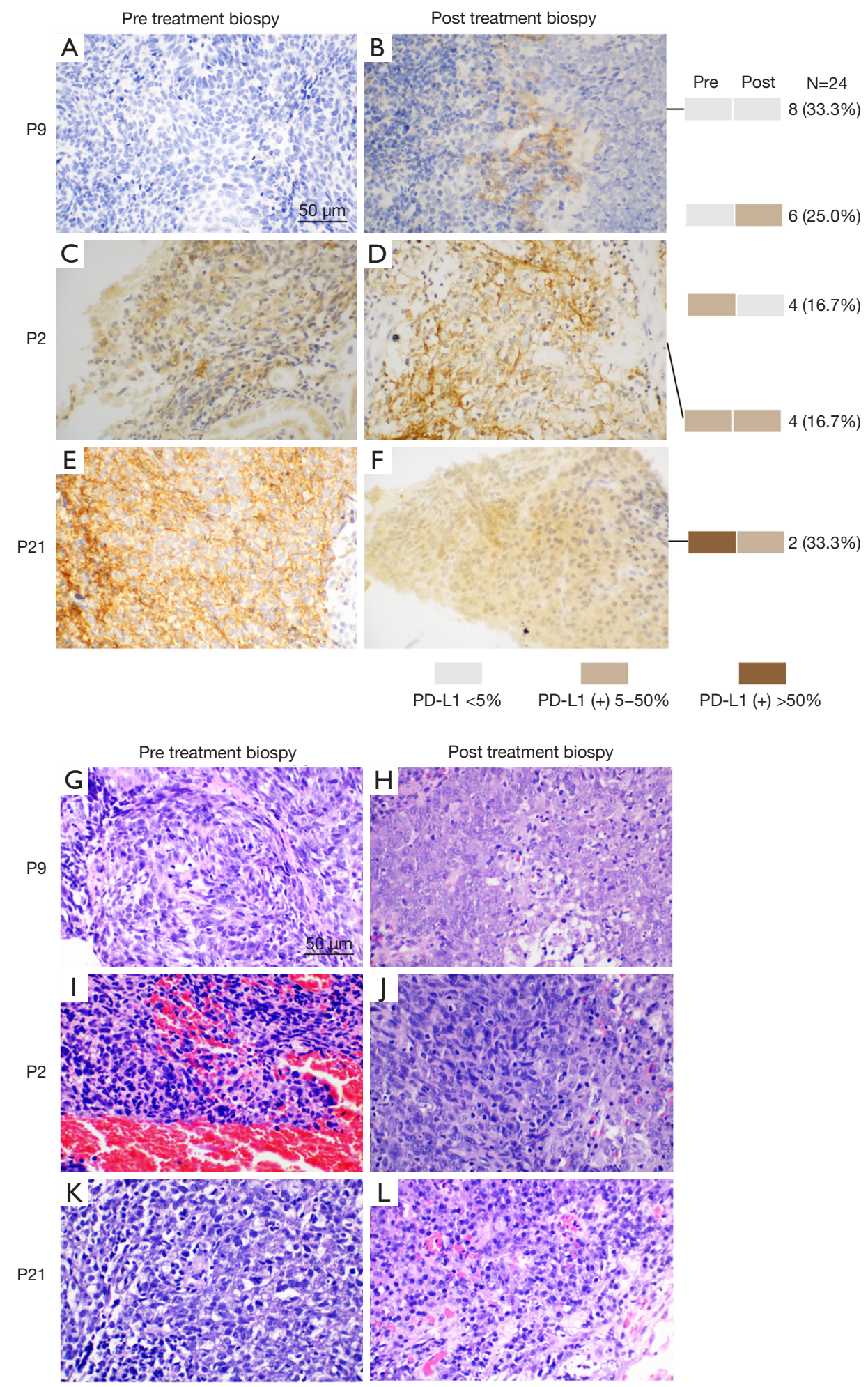

Figure 1 Pathological characteristics of advanced NPC patients. (A-F) PD-L1 immunochemistry staining of representative NPC biopsy pre/ post treatment; (G-L) H\&E staining of representative NPC biopsy pre/post treatment. Scale bar: $50 \mu \mathrm{m}$. NPC, nasopharyngeal carcinoma; PD-L1, programmed death-ligand 1. 
A Response to chemoradiotherapy

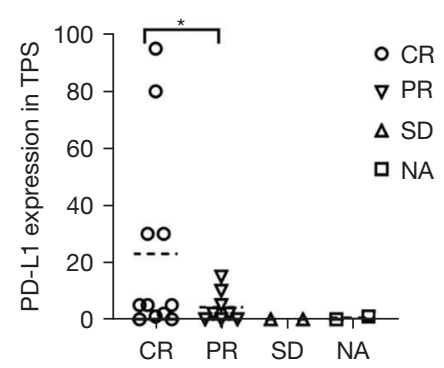

C

Alteration of PD-L1 expression in TPS

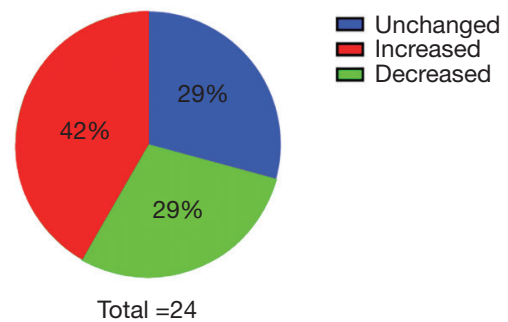

B Response to chemoradiotherapy

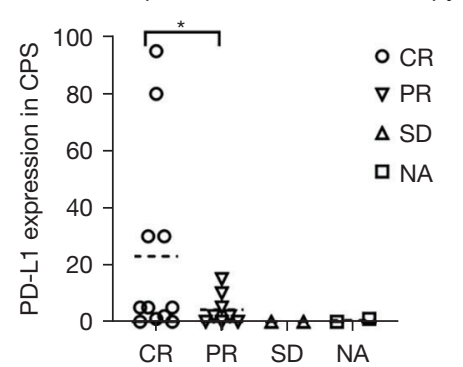

D

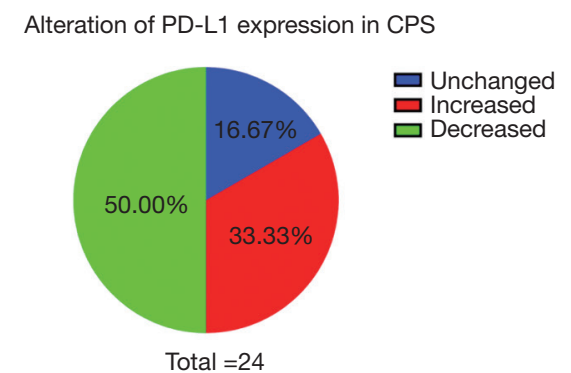

Figure 2 The landscape of PD-L1 expression alteration after concurrent chemoradiotherapy. (A) Correlation between PD-1 expression in TPS and patient response to chemoradiotherapy; (B) correlation between PD-1 expression in CPS and patient response to chemoradiotherapy; (C) alteration of PD-1 expression in TPS; (D) alteration of PD-1 expression in CPS. * $\mathrm{P}<0.05$. PD-L1, programmed death-ligand 1; TPS, tumor proportion score.

\section{Mutation spectrum}

The mutational spectra in our cohort confirmed that $\mathrm{C}>\mathrm{T}$ was the most common base substitution, followed by $\mathrm{C}>\mathrm{A}, \mathrm{T}>\mathrm{C}$, $\mathrm{C}>\mathrm{G}, \mathrm{T}>\mathrm{G}$, and $\mathrm{T}>\mathrm{A}$ (Figure 4A,4B). NPC samples exhibited a higher average $\mathrm{Ti}$ (transition) frequency of $52 \%$, compared to a $\mathrm{Tv}$ (transversion) frequency of $47 \%$ (Figure $4 C$ ). The distribution of mutations among each sample was significantly different; Figure $4 D$ comprehensively illustrates the distribution of mutations in 18 NPC samples. The missense mutation and single nucleotide polymorphisms (SNPs) accounted for the major mutation variants in our cohort (Figure 4E-4H). Except for patient 1 (P01), the total variants between each patient were similar.

\section{Discussion}

In the present study, we analyzed the immunological checkpoint PD-L1 expression alteration together with the clinical outcomes in locoregionally advanced NPC patients receiving CCRT. Our results indicated that NPC patients with higher PD-L1 scores (pre-treatment) had a significantly preferable clinical outcome. Hence, reassessing the PD-L1 expression after chemoradiotherapy for the best clinical benefit was recommended. Furthermore, nextgeneration sequencing and somatic mutation annotation demonstrated variants among the different NPC tumor biopsies, which may be largely attributable to the nature of tumor heterogeneity in NPC.

ICIs treatments have revolutionized cancer therapy in various types of cancers. Target the PD-1/PD-L1 pathway has shown to provide survival benefits in NPC patients $(24,25)$. The expression of PD-L1 has been reported as a prognostic predictor in different cancers, for NPC remains in dispute. Radiotherapy (RT) and chemotherapy are the main treatments for NPC (26). A recent study demonstrated that radiotherapy induced the expression of PD-L1 in NPC cells and PD-1 in NK cells, resulting in increased sensitivity of NPC cells to NK cell killing. Blocking of PD-L1/PD-1 checkpoint further increases the sensitivity of NPC cells to radiotherapy (27). In another study, PDL1 levels upregulated in NPC patients who received conventional chemoradiotherapy (21). However, Chan et al. demonstrated that PD-L1 expression of NPC patients 

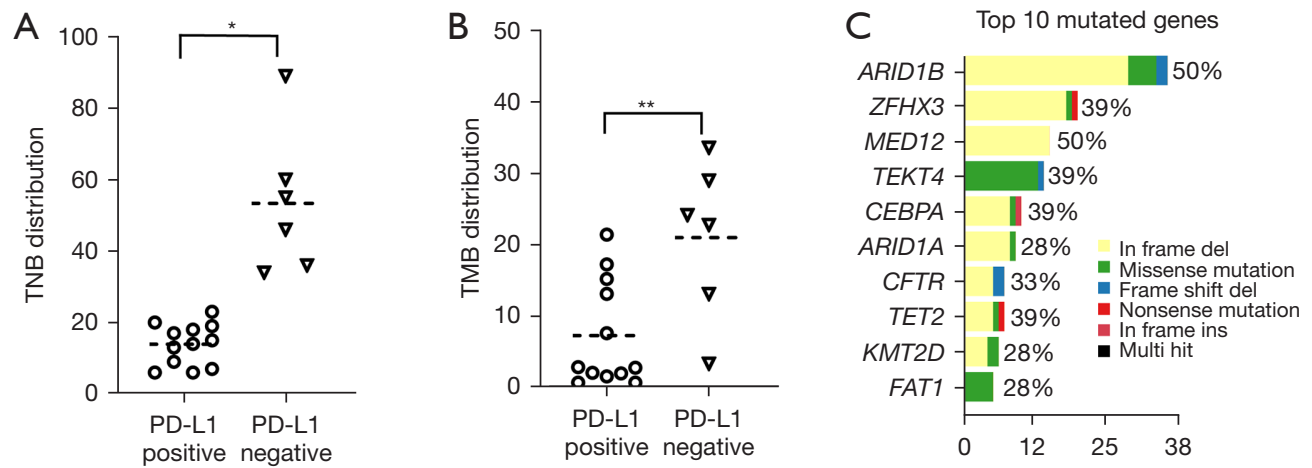

D

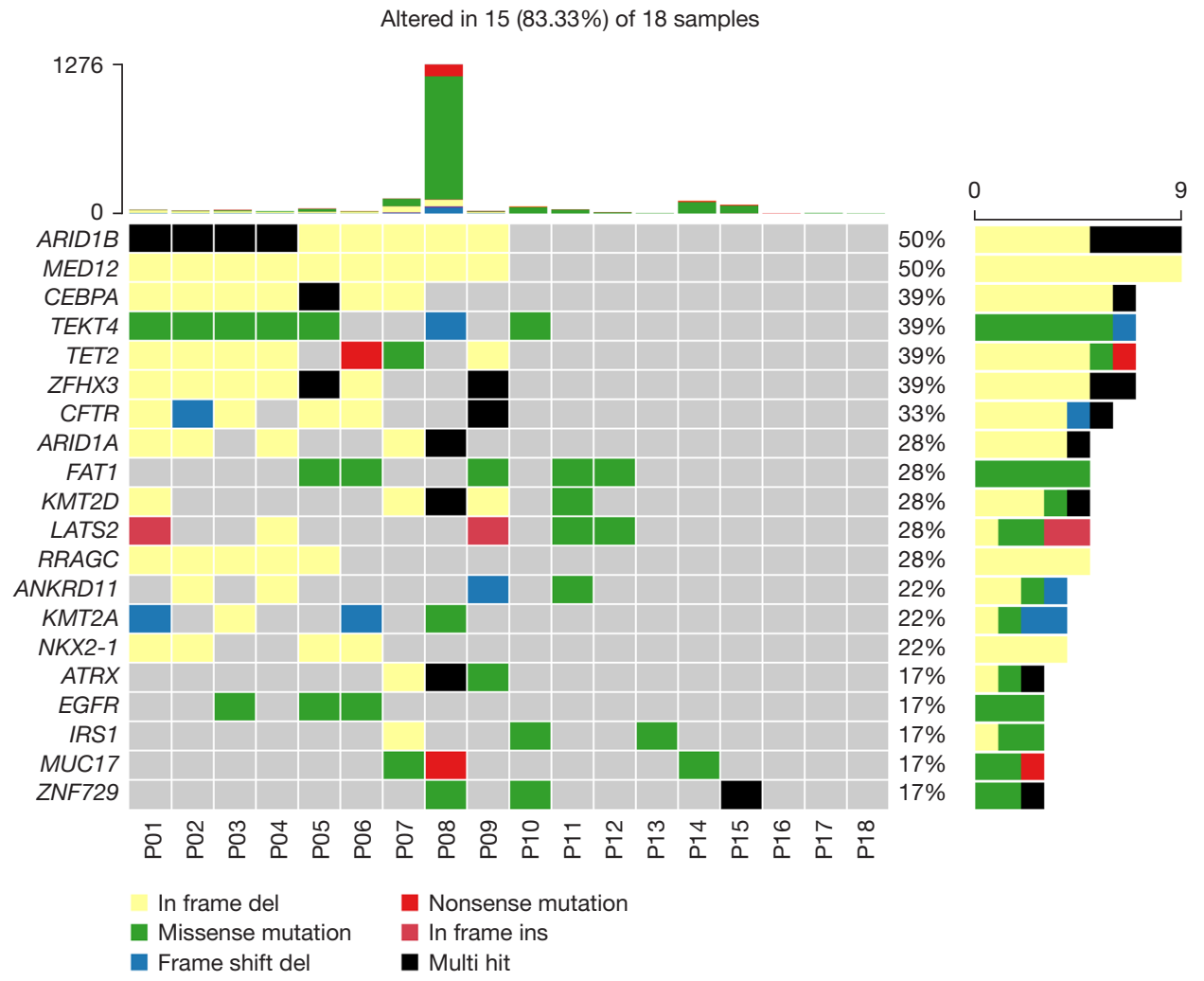

Figure 3 Landscape of somatic mutations of driver genes in NPC. (A) The TNB distribution in PD-L1 positive and negative groups; (B) the TMB distribution in PD-L1 positive and negative groups; (C) top mutated genes in NPC samples; (D) oncoprint of somatic mutations in 18 NPC samples. *, $\mathrm{P}<0.05$; **, $\mathrm{P}<0.01$. NPC, nasopharyngeal carcinoma; TNB, tumor neoantigen burden; PD-L1, programmed deathligand $1 ; \mathrm{TMB}$, tumor mutation burden.

reduced after treatment (radiotherapy with or without chemotherapy) (28). In this study, after CCRT, the PDL1 expression in TPS was unchanged in 7 patients (29\%), increased in 10 patients (42\%), and decreased in 7 patients (29\%). Interestingly, the PD-L1 expression in the CPS was unchanged in 4 patients (17\%), increased in 8 patients $(33 \%)$, and decreased in 12 patients (50\%). In addition, Cao et al. demonstrated that tumor PD-L1 expression is inversely associated with poor outcomes in patients with NPC (high PD-L1 expression is a poor prognostic factor 

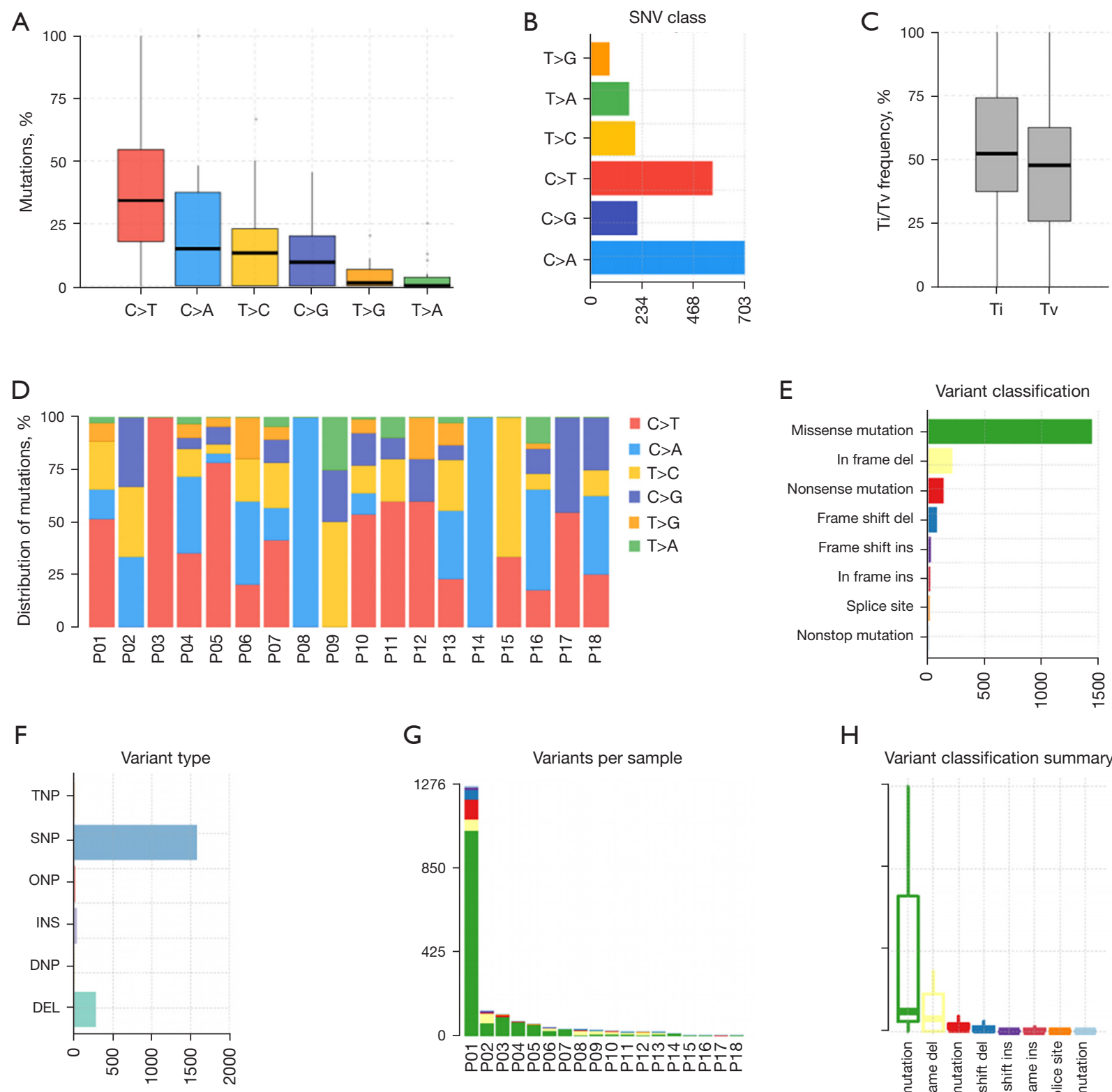

$\mathrm{H}$

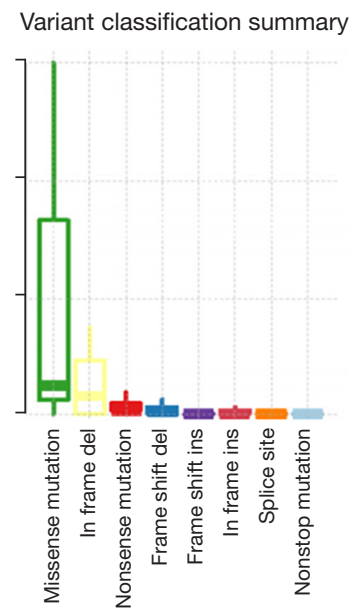

Figure 4 Mutation spectrum. (A) The distribution of base substitutions in all samples; (B) the Ti/Tv frequency in all samples; (C) mutation spectrum of 18 NPC samples in our cohort; (D) number of variants; (E) classification of variant; (F) SNV class; (G) variants per sample; (H) variant classification summary. Ti/Tv, transition/transversion; NPC, nasopharyngeal carcinoma; SNV, single nucleotide variant.

for NPC patients) (18). Inversely, Liu et al. reported that the OS of patients with high PD-L1 expression was longer than patients with low PD-L1 expression in NPC (29). Therefore, the correlation between PD-L1 expression and the outcomes of chemoradiotherapy has been controversial. In this study, we had not discussed this issue, and the correlation between PD-L1 and prognosis will investigate in subsequent studies. Notably, the mean values of the TMB and TNB in the PD-L1 positive group were significantly lower than the PD-L1 negative group in our cohort. Thus, TMB and TNB may be potential clinical indicators in NPC treatment. 
We verified 8,514 mutation events in 1,778 genes from cancer and adjacent tissues, including the most frequent driver gene mutations in NPC (TET2, TP53, and MAPK). These mutated genes were consistent with previous research depicting the potential biomarkers in NPC patients (30-32). Lin et al. identified 1,577 non-silent somatic mutations related to 1,413 genes, indicating that NPC has a relatively low mutational frequency and varying mutational diversity (33). Also, recent studies have suggested that crucial genomic alterations, such as TP53 and MAPK, stimulate the development and deterioration of NPC (34-36). Given that our research involved a small sample size, more NPC samples should be included in the future to assess the correlation between TMB and PD-L1 expression, and TNB and PD-L1 expression, and to obtain a more persuasive conclusion.

\section{Conclusions}

As immunotherapy is incorporated into standard treatment paradigms, optimized strategies and bio-predictors that predict the kinds of patients who could gain maximal benefit from immunotherapy are urgently needed. In the present study, we confirmed that the TMB and TNB may be potential clinical indicators in NPC treatment, and PD-L1 expression may be a clinical biomarker in NPC chemoradiotherapy. Through next-generation sequencing and annotation, we found that the most frequent driver gene mutations in NPC were TET2, TP53, and MAPK. We believe that our study could be instrumental for the identification of new genetic targets, which may provide new therapeutic strategies for the design of drug interventions that mitigate the severity of NPC, as well as the development of predictive biomarker responses to immunotherapy.

\section{Acknowledgments}

Funding: None.

\section{Footnote}

Reporting Checklist: The authors have completed the REMARK reporting checklist. Available at https://dx.doi. org/10.21037/atm-21-5175

Data Sharing Statement: Available at https://dx.doi. org/10.21037/atm-21-5175
Conflicts of Interest: All authors have completed the ICMJE uniform disclosure form (available at https://dx.doi. org/10.21037/atm-21-5175). The authors have no conflicts of interest to declare.

Etbical Statement: The authors are accountable for all aspects of the work in ensuring that questions related to the accuracy or integrity of any part of the work are appropriately investigated and resolved. All procedures performed in this study involving human participants were under the Declaration of Helsinki (revised in 2013). This study was approved by the Ethics Committee of Tongji Hospital of Tongji Medical College, Huazhong University of Science and Technology, China. All methods were carried out their relevant guidelines and regulations. Written informed consent was obtained from all study participants.

Open Access Statement: This is an Open Access article distributed in accordance with the Creative Commons Attribution-NonCommercial-NoDerivs 4.0 International License (CC BY-NC-ND 4.0), which permits the noncommercial replication and distribution of the article with the strict proviso that no changes or edits are made and the original work is properly cited (including links to both the formal publication through the relevant DOI and the license). See: https://creativecommons.org/licenses/by-nc-nd/4.0/.

\section{References}

1. Carioli G, Negri E, Kawakita D, et al. Global trends in nasopharyngeal cancer mortality since 1970 and predictions for 2020: Focus on low-risk areas. Int J Cancer 2017;140:2256-64.

2. Chen YP, Chan ATC, Le QT, et al. Nasopharyngeal carcinoma. Lancet 2019;394:64-80.

3. Lin C, Lin S, Guo QJ, et al. Systemic immuneinflammation index as a prognostic marker in patients with newly diagnosed metastatic nasopharyngeal carcinoma: a propensity score-matched study. Transl Cancer Res 2019;8:2089-98.

4. Lee AW, Ma BB, Ng WT, et al. Management of Nasopharyngeal Carcinoma: Current Practice and Future Perspective. J Clin Oncol 2015;33:3356-64.

5. Kang $\mathrm{Y}, \mathrm{He} \mathrm{W}$, Ren C, et al. Advances in targeted therapy mainly based on signal pathways for nasopharyngeal carcinoma. Signal Transduct Target Ther 2020;5:245.

6. Guan S, Wei J, Huang L, et al. Chemotherapy and chemo- 
resistance in nasopharyngeal carcinoma. Eur J Med Chem 2020;207:112758.

7. Tang LL, Chen WQ, Xue WQ, et al. Global trends in incidence and mortality of nasopharyngeal carcinoma. Cancer Lett 2016;374:22-30.

8. Tang LQ, Chen DP, Guo L, et al. Concurrent chemoradiotherapy with nedaplatin versus cisplatin in stage II-IVB nasopharyngeal carcinoma: an open-label, non-inferiority, randomised phase 3 trial. Lancet Oncol 2018;19:461-73.

9. Huang ZL, Liu S, Wang GN, et al. The prognostic significance of PD-L1 and PD-1 expression in patients with nasopharyngeal carcinoma: a systematic review and meta-analysis. Cancer Cell Int 2019;19:141.

10. Eichberger J, Schulz D, Pscheidl K, et al. PD-L1 Influences Cell Spreading, Migration and Invasion in Head and Neck Cancer Cells. Int J Mol Sci 2020;21:8089.

11. Jing $W$, Li M, Zhang Y, et al. PD-1/PD-L1 blockades in non-small-cell lung cancer therapy. Onco Targets Ther 2016;9:489-502.

12. Federico P, Petrillo A, Giordano P, et al. Immune Checkpoint Inhibitors in Hepatocellular Carcinoma: Current Status and Novel Perspectives. Cancers (Basel) 2020;12:3025.

13. Liu X, Shan C, Song Y, et al. Prognostic Value of Programmed Cell Death Ligand-1 Expression in Nasopharyngeal Carcinoma: A Meta-Analysis of 1,315 Patients. Front Oncol 2019;9:1111.

14. Pötzsch M, Berg E, Hummel M, et al. Better prognosis of gastric cancer patients with high levels of tumor infiltrating lymphocytes is counteracted by PD-1 expression. Oncoimmunology 2020;9:1824632.

15. Zhao X, Kong Y, Zhang L. Anti-PD-1 Immunotherapy Combined With Stereotactic Body Radiation Therapy and GM-CSF as Salvage Therapy in a PD-L1-Negative Patient With Refractory Metastatic Esophageal Squamous Cell Carcinoma: A Case Report and Literature Review. Front Oncol 2020;10:1625.

16. Stovgaard ES, Dyhl-Polk A, Roslind A, et al. PD-L1 expression in breast cancer: expression in subtypes and prognostic significance: a systematic review. Breast Cancer Res Treat 2019;174:571-84.

17. Takada K, Toyokawa G, Kinoshita F, et al. Expression of PD-L1, PD-L2, and IDO1 on tumor cells and density of CD8-positive tumor-infiltrating lymphocytes in early-stage lung adenocarcinoma according to histological subtype. J Cancer Res Clin Oncol 2020;146:2639-50.
18. Cao Y, Chan KI, Xiao G, et al. Expression and clinical significance of PD-L1 and BRAF expression in nasopharyngeal carcinoma. BMC Cancer 2019;19:1022.

19. Kawaguchi T, Ono T, Sato F, et al. CD8+ T Cell Infiltration Predicts Chemoradiosensitivity in Nasopharyngeal or Oropharyngeal Cancer. Laryngoscope 2021;131:E1179-E1189.

20. Takamori S, Takada K, Azuma K, et al. Prognostic Impact of Programmed Death-Ligand 2 Expression in Primary Lung Adenocarcinoma Patients. Ann Surg Oncol 2019;26:1916-24.

21. Zhang J, Fang W, Qin T, et al. Co-expression of PD-1 and PD-L1 predicts poor outcome in nasopharyngeal carcinoma. Med Oncol 2015;32:86.

22. McKenna A, Hanna M, Banks E, et al. The Genome Analysis Toolkit: a MapReduce framework for analyzing next-generation DNA sequencing data. Genome Res 2010;20:1297-303.

23. Talevich E, Shain AH, Botton T, et al. CNVkit: GenomeWide Copy Number Detection and Visualization from Targeted DNA Sequencing. PLoS Comput Biol 2016;12:e1004873.

24. Chow JC, Ngan RK, Cheung KM, et al. Immunotherapeutic approaches in nasopharyngeal carcinoma. Expert Opin Biol Ther 2019;19:1165-72.

25. Jain A, Chia WK, Toh HC. Immunotherapy for nasopharyngeal cancer-a review. Chin Clin Oncol 2016;5:22.

26. Tsang J, Lee VH, Kwong DL. Novel therapy for nasopharyngeal carcinoma--where are we. Oral Oncol 2014;50:798-801.

27. Makowska A, Lelabi N, Nothbaum C, et al. Radiotherapy Combined with PD-1 Inhibition Increases NK Cell Cytotoxicity towards Nasopharyngeal Carcinoma Cells. Cells 2021;10:2458.

28. Chan OS, Kowanetz M, Ng WT, et al. Characterization of PD-L1 expression and immune cell infiltration in nasopharyngeal cancer. Oral Oncol 2017;67:52-60.

29. Liu YJ, Tsang NM, Hsueh C, et al. Low PD-L1 Expression Strongly Correlates with Local Recurrence in Epstein-Barr Virus-Positive Nasopharyngeal Carcinoma after Radiation-Based Therapy. Cancers (Basel) 2018;10:374.

30. Cho WCS, Tse KP, Ngan RKC, et al. Genomic characterization reveals potential biomarkers in nasopharyngeal carcinoma patients with relapse. Expert Rev Mol Diagn 2020;20:1149-59.

31. Li Z, Fu J, Li N, et al. Quantitative proteome analysis 
identifies MAP2K6 as potential regulator of LIFRinduced radioresistance in nasopharyngeal carcinoma cells. Biochem Biophys Res Commun 2018;505:274-81.

32. Zhang X, Yang J, Shi D, et al. TET2 suppresses nasopharyngeal carcinoma progression by inhibiting glycolysis metabolism. Cancer Cell Int 2020;20:363.

33. Lin DC, Meng X, Hazawa M, et al. The genomic landscape of nasopharyngeal carcinoma. Nat Genet 2014;46:866-71.

34. Zhang L, MacIsaac KD, Zhou T, et al. Genomic Analysis of Nasopharyngeal Carcinoma Reveals TME-Based

Cite this article as: Long G, Li X, Yang L, Zhao J, Lu X, Wang H, Song J, Mei Q, Hu G. The clinical prognostic value of PD-L1 after concurrent chemoradiotherapy in Chinese nasopharyngeal carcinoma patients. Ann Transl Med 2021;9(22):1650. doi: 10.21037/atm-21-5175
Subtypes. Mol Cancer Res 2017;15:1722-32.

35. Zhang Z, Yu X, Zhou Z, et al. LMP1-positive extracellular vesicles promote radioresistance in nasopharyngeal carcinoma cells through P38 MAPK signaling. Cancer Med 2019;8:6082-94.

36. Lin X, Bi Z, Hu Q, et al. TSPAN8 serves as a prognostic marker involving Akt/MAPK pathway in nasopharyngeal carcinoma. Ann Transl Med 2019;7:470.

(English Language Editor: A. Kassem) 
Table S1 Patients characteristic of 24 NPC patients

\begin{tabular}{|c|c|c|c|c|c|c|c|c|c|c|c|c|c|c|c|c|}
\hline \multirow{2}{*}{$\begin{array}{l}\text { Patient } \\
\text { name }\end{array}$} & \multirow{2}{*}{ Patient ID } & \multirow{2}{*}{ Gender } & \multirow{2}{*}{$\begin{array}{l}\text { Age at } \\
\text { diagnosis } \\
\text { (yrs) }\end{array}$} & \multirow{2}{*}{$\begin{array}{l}\text { Induction chemotherapy } \\
\text { regimen }\end{array}$} & \multirow{2}{*}{$\begin{array}{l}\text { Radiotherapy } \\
\text { regime }\end{array}$} & \multirow{2}{*}{$\begin{array}{l}\text { Pathological } \\
\text { TNM }\end{array}$} & \multirow{2}{*}{$\begin{array}{l}\text { Clinical } \\
\text { stage }\end{array}$} & \multirow{2}{*}{ TNB } & \multirow{2}{*}{ TMB } & \multicolumn{2}{|c|}{ TPS } & \multicolumn{2}{|c|}{ CPS } & \multicolumn{2}{|c|}{$\begin{array}{l}\text { EB virus } \\
\text { (copies/mL) }\end{array}$} & \multirow{2}{*}{ Response } \\
\hline & & & & & & & & & & Pre & Post & Pre & Post & Pre & Post & \\
\hline ads & $\mathrm{P} 1$ & Female & 46 & $\mathrm{DOC}+\mathrm{NDP}+5 \mathrm{FU}$ & IMRT & T4N1 & IVA & 23 & 24.14 & $2 \%$ & $10 \%$ & $2 \%$ & $12 \%$ & $<500$ & 800 & PR \\
\hline $\operatorname{ccs}$ & P2 & Male & 54 & $\mathrm{DOC}+\mathrm{NDP}+5 \mathrm{FU}$ & IMRT & T4N2 & IVA & 15 & 22.47 & $30 \%$ & $50 \%$ & $70 \%$ & $55 \%$ & 4250 & - & $\mathrm{CR}$ \\
\hline $\mathrm{ch}$ & P3 & Female & 49 & $\mathrm{DOC}+\mathrm{NDP}+5 \mathrm{FU}$ & IMRT & T4N1 & IVA & 46 & 1.57 & $10 \%$ & - & - & - & 500 & 1350 & PR \\
\hline cxl & P4 & Male & 46 & $\mathrm{DOC}+\mathrm{NDP}+5 \mathrm{FU}$ & IMRT & T4N2 & IVA & 20 & 22.76 & - & - & $5 \%$ & - & 1600 & 2250 & PR \\
\hline hjh & P5 & Male & 52 & $\mathrm{DOC}+\mathrm{DDP}+\mathrm{CAP}$ & IMRT & $\mathrm{T} 4 \mathrm{~N} 1$ & IVA & 15 & 17.24 & $2 \%$ & - & $7 \%$ & - & - & - & $\mathrm{CR}$ \\
\hline jxl & P6 & Male & 45 & $\mathrm{DOC}+\mathrm{NDP}+5 \mathrm{FU}$ & IMRT & T2N3 & IVA & 19 & 21.38 & $5 \%$ & - & $5 \%$ & - & 1850 & - & PR \\
\hline lj & P7 & Male & 41 & $\mathrm{DOC}+\mathrm{NDP}+5 \mathrm{FU}$ & IMRT & T3N1 & III & 6 & 7.59 & - & - & - & - & 1350 & - & CR \\
\hline Ich & P8 & Male & 54 & $\mathrm{DOC}+\mathrm{NDP}$ & IMRT & T4N2 & IVA & 2 & 2.75 & $1 \%$ & $30 \%$ & $2 \%$ & $30 \%$ & 1000 & - & NA \\
\hline myl & P9 & Male & 52 & $\mathrm{DOC}+\mathrm{NDP}+5 \mathrm{FU}$ & IMRT & T3N1 & III & 89 & 2.84 & - & $5 \%$ & - & $5 \%$ & - & - & PR \\
\hline qgx & P10 & Female & 45 & $\mathrm{DOC}+\mathrm{NDP}+5 \mathrm{FU}$ & IMRT & T3N2 & III & 55 & 1.97 & $5 \%$ & - & $20 \%$ & - & 1150 & - & CR \\
\hline sj & P11 & Female & 30 & $\mathrm{DOC}+\mathrm{NDP}$ & IMRT & T3NO & III & / & / & $5 \%$ & $20 \%$ & $55 \%$ & $25 \%$ & $<500$ & - & $\mathrm{CR}$ \\
\hline wyl & P12 & Female & 41 & $\mathrm{DOC}+\mathrm{NDP}$ & IMRT & $\mathrm{T} 4 \mathrm{~N} 1$ & IVA & I & / & $1 \%$ & $20 \%$ & $2 \%$ & $40 \%$ & - & - & $\mathrm{CR}$ \\
\hline wb & $\mathrm{P} 13$ & Male & 40 & $\mathrm{DOC}+\mathrm{DDP}+\mathrm{CAP}$ & IMRT & T4N2 & IVA & 7 & 13.11 & - & - & $5 \%$ & $2 \%$ & 3450 & $<500$ & PR \\
\hline wkx & P14 & Male & 43 & $\mathrm{DOC}+\mathrm{NDP}$ & IMRT & T3N3 & IVA & 60 & 3.37 & - & - & - & - & & & / \\
\hline wl & P15 & Male & 51 & $\mathrm{DOC}+\mathrm{NDP}+5 \mathrm{FU}$ & IMRT & T3N3 & IVA & / & / & - & $2 \%$ & $1 \%$ & $5 \%$ & - & - & $\mathrm{CR}$ \\
\hline$x w x$ & P16 & Female & 44 & $\mathrm{DOC}+\mathrm{NDP}+5 \mathrm{FU}$ & IMRT & T4N2 & IVA & / & / & - & $50 \%$ & $1 \%$ & $50 \%$ & - & - & $\mathrm{CR}$ \\
\hline xhf & P17 & Male & 58 & $\mathrm{DOC}+\mathrm{DDP}+5 \mathrm{FU}$ & IMRT & T4N2 & IVA & / & / & $15 \%$ & $30 \%$ & $20 \%$ & $32 \%$ & $<500$ & / & PR \\
\hline yzc & P18 & Male & 47 & $\mathrm{DOC}+\mathrm{NDP}+\mathrm{CAP}$ & IMRT & T3N3 & IVA & / & / & $2 \%$ & - & $3 \%$ & - & $<500$ & - & PR \\
\hline yls & P19 & Male & 56 & GEM + DDP & IMRT & T4N2 & IVA & 27 & 35.14 & - & - & $2 \%$ & - & $<500$ & 850 & SD \\
\hline zc & P20 & Male & 48 & $\mathrm{DOC}+\mathrm{NDP}+5 \mathrm{FU}$ & IMRT & T3N2 & III & / & / & - & $10 \%$ & $2 \%$ & $10 \%$ & / & / & NA \\
\hline zsq & P21 & Male & 47 & $\mathrm{DOC}+\mathrm{NDP}$ & IMRT & T1N1 & II & I & / & $80 \%$ & $50 \%$ & $81 \%$ & $51 \%$ & 2050 & - & $\mathrm{CR}$ \\
\hline zjx & P22 & Male & 19 & $\mathrm{DOC}+\mathrm{NDP}+5 \mathrm{FU}$ & IMRT & T4N2 & IVA & / & / & $95 \%$ & $40 \%$ & $95 \%$ & $40 \%$ & - & - & CR \\
\hline zqm & P23 & Male & 64 & GEM + DDP & IMRT & T3N2 & III & 43 & 41.64 & - & - & $1 \%$ & - & - & - & SD \\
\hline cyq & P24 & Male & 55 & $\mathrm{DOC}+\mathrm{NDP}+5 \mathrm{FU}$ & IMRT & T3N3 & III & 3 & 2.07 & $5 \%$ & $5 \%$ & $6 \%$ & $6 \%$ & 1500 & - & $\mathrm{CR}$ \\
\hline
\end{tabular}

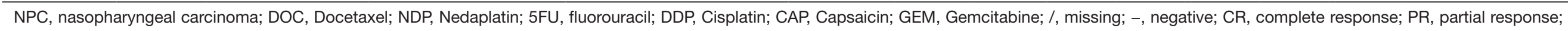
NR, no response; NA, not available. 